\title{
Uso de embalagens associadas às práticas de consumo alimentar na pandemia SARS-
} CoV-2

\author{
Use of packaging associated with food consumption practices in the SARS-CoV-2 \\ Uso de empaques asociados a prácticas de consumo de alimentos en la pandemia SARS-CoV-2
}

Recebido: 09/02/2022 | Revisado: 18/02/2022 | Aceito: 21/02/2022 | Publicado: 03/03/2022

Rodrigo Rossetti Veloso

ORCID: https://orcid.org/0000-0002-0510-0882 Instituto Federal de Pernambuco, Brasil E-mail: rodrigo.rossetti@cabo.ifpe.edu.br

Aline Gomes Santana

ORCID: https://orcid.org/0000-0002-8432-5149

Universidade Federal Rural de Pernambuco, Brasil E-mail: Alinegsan157@gmail.com

Gabriela Ferreira Rodrigues

ORCID: https://orcid.org/0000-0002-1780-0579

Universidade Federal Rural de Pernambuco, Brasil

E-mail: gabriela.frodriguess@gmail.com

João Pedro Correia Lacerda

ORCID: https://orcid.org/0000-0003-0057-228X

Universidade Federal Rural de Pernambuco, Brasil

E-mail: jpedrinho.correia@gmail.com

Izabel Cristina Silva Amorim

ORCID: https://orcid.org/0000-0001-8214-999X

Universidade Federal Rural de Pernambuco, Brasil E-mail: bel.gastronomiapt@gmail.com

Gisele Estevão de Lima

ORCID: https://orcid.org/0000-0003-1901-8078 Instituto Federal de Pernambuco, Brasil E-mail: Giselleestevao@gmail.com

Neide Kazue Sakugawa Shinohara

ORCID: https://orcid.org/0000-0001-8356-874X

Universidade Federal Rural de Pernambuco, Brasil E-mail: neide.shinohara@ufrpe.br

\begin{abstract}
Resumo
A pandemia provocada por SARS-CoV-2 (Covid-19) impôs o fechamento comercial e restrição das atividades laborais no Brasil e em vários países do globo. Essa nova realidade modificou e alavancou o modelo de comercialização de alimentos prontos para o consumo, baseado em embalagens e utensílios descartáveis. Foi realizado um estudo transversal descritivo, cujo objetivo foi a aplicação de formulário virtual direcionado a empreendimentos gastronômicos, para estudar as embalagens e a logística de entrega de alimentos prontos no período pandêmico nos estados de Alagoas, Minas Gerais, Pará, Pernambuco, Rio de Janeiro e São Paulo. O modelo delivery por aplicativo foi o mais frequente como instrumento de prática de consumo, usando como materiais de embalagens: plástico (65,9\%), isopor (48,8\%); papel cartonado (39\%) e alumínio (30,9\%). Esses achados são preocupantes, pois com exceção do papel, o restante dos materiais empregados, representam fortes poluentes ambientes, devido à dificuldade de decomposição natural e uso de tecnologia onerosa de mitigação.
\end{abstract}

Palavras-chave: Pandemia Covid-19; Consumo de alimentos; Embalagem para alimentos.

\begin{abstract}
The pandemic caused by SARS-CoV-2 (Covid-19) imposed the commercial closure and restriction of work activities in Brazil and in several countries around the world. This new reality has modified and leveraged the ready-to-eat food sales model, based on disposable packaging and utensils. A descriptive cross-sectional study was carried out, whose objective was the application of a virtual form directed to gastronomic enterprises, to study the packing and logistics of delivery of ready-to-eat foods during the pandemic period in the states of Alagoas, Minas Gerais, Pará, Pernambuco, Rio de Janeiro, and São Paulo. The delivery model by application was the most frequent as an instrument of consumption practice, using as packaging materials: plastic (65.9\%), Styrofoam (48.8\%); cardboard $(39 \%)$ and aluminum (30.9\%). These findings are worrying because, except for paper, the rest of the materials used
\end{abstract}


represent strong environmental pollutants, due to the difficulty of natural decomposition and the use of costly mitigation technology.

Keywords: Covid-19 pandemic; Food consumption; Food packaging.

\section{Resumen}

La pandemia provocada por el SARS-CoV-2 (Covid-19) impuso el cierre comercial y la restricción de actividades laborales en Brasil y en varios países del mundo. Esta nueva realidad ha modificado y potenciado el modelo de venta de alimentos listos para el consumo, basado en envases y utensilios desechables. Se realizó un estudio descriptivo de corte transversal, cuyo objetivo fue la aplicación de un formulario virtual dirigido a empresas gastronómicas, para estudiar el empaque y la logística de entrega de alimentos listos para el consumo durante el período de pandemia en los estados de Alagoas, Minas Gerais, Pará, Pernambuco, Río de Janeiro y São Paulo. El modelo de entrega por aplicación fue el más frecuente como instrumento de práctica de consumo, utilizando como materiales de embalaje: plástico (65,9\%), poliestireno (48,8\%); cartón (39\%) y aluminio (30,9\%). Estos hallazgos son preocupantes porque, con excepción del papel, el resto de los materiales utilizados representan fuertes contaminantes ambientales, debido a la dificultad de descomposición natural y al uso de costosas tecnologías de mitigación.

Palabras clave: Pandemia de COVID-19; Consumo de comida; Envasado de alimentos.

\section{Introdução}

Desde o fim de 2019, o mundo tem sido assolado pela rápida disseminação do novo coronavírus, denominado SARSCoV-2, nomenclatura adotada mundialmente advinda da relação entre esse agente e o desenvolvimento de síndrome respiratória aguda grave, porque essa classe de vírus tem afinidade e capacidade invasiva pela árvore respiratória (Moreira et al., 2022). A pandemia fez com que as pessoas passassem a se sentir mais seguras em casa, e isto resultou em modificações nos padrões de consumo de alimentos, principalmente no que diz respeito a compras pela internet, através de aplicativos de entreg a de comida pronta. Este novo cenário refletiu diretamente na produção e posteriormente no descarte substancial de diversos produtos descartáveis, como por exemplo, recipientes plásticos para comidas e bebidas, talheres e sacos plásticos (Teixeira \& Mourão, 2021).

Nesse período de pandemia global, com normas sanitárias de distanciamento social, os restaurantes tiveram que se adaptar a uma nova rotina alimentar, principalmente na questão do uso de embalagem descartável e envio dos alimentos prontos para consumo seguro. Segundo Brasil (2004), a resolução que trata do Regulamento Técnico de Boas Práticas para Serviços de Alimentação, tem como objetivo garantir as condições higiênico-sanitárias do alimento preparado pelas unidades de alimentação, que com a chegada dessa pandemia, acabou provocando modificações da logística de produção, que vão desde a garantia da qualidade da matéria prima à execução dos serviços prestados, principalmente na segurança sanitária da manipulação e a forma segura de entrega desses alimentos.

Com o fechamento obrigatório dos restaurantes, no chamado período de lockdown, definido segundo cada gestor público, os consumidores poderiam receber as preparações nos locais previamente determinados, não sendo permitido o consumo nos espaços dos serviços de alimentação. Assim, os produtores de refeição e os responsáveis técnicos tiveram que estudar novos meios de entrega, para que o seu produto chegasse na ponta final, que é o cliente, de forma rápida, segura, sem avarias e/ou que não houvesse exposição do alimento com o ambiente externo, situação que seria passível de contaminação microbiológica cruzada.

Com o passar dos anos, os serviços de entrega ganharam espaço ao redor do mundo, expandindo para diversos mercados como o de supermercados, o farmacêutico, têxtil, livraria, eletroeletrônico e o gastronômico. Esse tipo de serviço engloba algumas modalidades como o delivery, drive-thru e a retirada no local, formas de comercialização que se aperfeiçoaram com o tempo e a percepção do consumidor.

Segundo Dutra e Zani (2020), o delivery, diferente do que muitos imaginam, tem suas origens na Roma antiga, onde era um serviço necessário para parte da população que não tinha condições econômicas de ter uma cozinha em casa. Tal prática pode ser definida como a entrega de um determinado produto a um local indicado pelo cliente, podendo ser, por exemplo, em 
casa ou no trabalho. Hoje em dia, com o desenvolvimento dos meios de transportes e das tecnologias, o serviço de delivery não é mais o mesmo de séculos atrás. A retirada no local, conhecida também com take-away, é um sistema em que o cliente faz o pedido previamente e se desloca até o estabelecimento para retirar seu produto quando estiver pronto. Desse modo, esse serviço não necessita do trabalho de um entregador, que se tem no delivery e, diferente do drive-thru, não é preciso esperar no local enquanto seu pedido está sendo feito. O take-away é um modelo que une a comodidade do delivery com a conveniência do drive-thru (Ruggiero, 2021).

Diante desta nova realidade da crise sanitária mundial, alguns serviços na área da alimentação, começaram suas atividades de entrega sem uma previsão de como seriam os desafios e consequências que enfrentariam: prolongada ausência dos clientes, redução da margem de lucratividade nas preparações transportadas, adaptação de mão de obra à nova realidade de entrega e oscilação dos preços das embalagens.

No mundo moderno, as práticas de produção sustentável são parte do contexto da ética alimentar, envolvendo cuidados com o meio ambiente, formas justas de comercialização e uso de embalagem reciclável ou retornável. A aplicação destas práticas cabe aos cientistas, empresários e gestores das políticas públicas, que precisam desempenhar um papel cada vez mais participativo, e buscando solução para os problemas de aplicação destas práticas, sempre alinhados com o desenvolvimento econômico e proteção das futuras gerações (Campbell- Platt, 2015).

Um dos principais impactos da pandemia aconteceu na esfera ambiental, onde segundo a Associação brasileira de empresas de limpeza pública e resíduos especiais (Abrelpe) em pesquisa divulgada em 2021, ocorreu um aumento de $25 \%$ do uso de plástico, durante os meses iniciais da pandemia, decorrentes da alta demanda de processos e práticas de consumo permitida pelos órgãos fiscalizadores no Brasil.

Deste modo, o estudo realizado neste artigo teve como objetivo estudar as embalagens e a logística de entrega de alimentos prontos no período pandêmico nos estados de Alagoas, Minas Gerais, Pará, Pernambuco, Rio de Janeiro e São Paulo. Verificando a percepção e o comportamento dos empreendedores quanto à seleção e aquisição de embalagens para entrega de comida, visando atender as determinações sanitárias e ao mesmo tempo satisfazer os clientes.

\section{Metodologia}

A fim de compreender o cenário pandêmico, foi realizado um estudo transversal descritivo, visto que permite, segundo Gil (2002), analisar e expor as características de um determinado grupo relacionando-as com as variáveis previamente estabelecidas. Juntamente a isso, o estudo também faz um levantamento bibliográfico, a fim de apresentar as diversas perspectivas que envolvem o tema. Desse modo, a técnica aplicada foi o questionário, que possibilitou coletar dados de uma forma mais rápida e segura, além de não exigir treinamento de pessoal e garantir o anonimato. A aplicação de formulários eletrônicos, além de ser econômica e ambientalmente viável, é assertiva e eficaz em relação à aplicação presencial, possibilitando atingir um público maior porque é enviado remotamente (Novaes et al., 2020).

A pesquisa foi direcionada a 110 proprietários de empreendimentos gastronômicos atuantes nos estados de Alagoas, Minas Gerais, Pará, Pernambuco, Rio de Janeiro e São Paulo, sendo disponibilizado através das redes sociais, realizando o convite para participação de forma espontânea, sem a exigência de dados pessoais ou que pudessem comprometer o entrevistado e/ou seu empreendimento, preservando o sigilo dos participantes.

O questionário que foi criado pelos autores possui 21 questões, a maioria era de múltipla escolha, visando a facilidade e a rapidez das questões. Inicialmente, buscou-se adquirir informações sobre o serviço de alimentação com 5 questões, com objetivo de caracterizá-lo. Destacamos, que nesta parte era opcional informar a localização do estabelecimento, ficando à critério do entrevistado responder ou declinar a resposta. Em seguida, questões para melhor entendimento sobre a logística de entrega, suas modalidades e os respectivos impactos durante a pandemia com 3 questões. E, para finalizar, perguntamos acerca 
das embalagens utilizadas no delivery/retirada no local, com 13 questões, para melhor elucidar os desafios, dificuldades, em momento de crise sanitária mundial.

\section{Resultados e Discussão}

Diante do cenário da pandemia do novo coronavírus, as organizações de saúde impuseram medidas de isolamento social a fim de conter a disseminação do vírus. Tais normas impactaram diversos empreendimentos, dentre eles os gastronômicos, que tiveram que reinventar o modo de comercializar seu produto. Com a impossibilidade de reunir os clientes no próprio negócio, estabelecimentos do ramo alimentício encontraram nos serviços de entrega um meio de manter a empresa funcionando. Com a implementação dos serviços de entrega foi necessário dar uma atenção e cuidado maior às embalagens que são utilizadas para comportar os alimentos. Deve-se levar em consideração que o tipo de embalagem escolhido pelo estabelecimento tem que manter a temperatura e garantir que o alimento chegue íntegro ao cliente, sem que a composição da embalagem possa interagir com o alimento acondicionado.

O contexto de pandemia, trouxe diversas mudanças para o mercado gastronômico, que teve que se adaptar às novas formas de consumo. Dentre os desafios enfrentados pelos empreendimentos desse setor tem-se a inclusão de um ou mais tipos de serviços de entrega, a análise da logística desse modelo e um estudo de qual a embalagem ideal para melhor atender a clientela.

O questionário foi respondido por 110 proprietários de empreendimentos gastronômicos e a partir das primeiras seções do formulário foi possível analisar que os entrevistados se distribuem em 6 estados brasileiros: Alagoas, Minas Gerais, Pará, Pernambuco, Rio de Janeiro e São Paulo. Observa-se que, de quando aplicado o questionário, nos meses de abril e maio de 2021, o mundo já estava com mais de um ano de convívio com a pandemia e 32,5\% dos estabelecimentos disseram ter um ano ou menos de funcionamento. Tal movimento é amparado pelo fato do fechamento de diversos empreendimentos e pela demissão ou ociosidade de diversos setores durante a pandemia, que possivelmente fez com que outros setores se voltassem para o setor de alimentação, como uma tentativa de sobreviver a este momento ou mesmo como uma oportunidade de negócio. Outro fator importante é que 65\% dos entrevistados afirmaram ter apenas uma unidade do estabelecimento e 18,7\% afirmaram funcionar apenas digitalmente na modalidade delivery, número que cresceu no período de pandemia. Segundo a ABRASEL (2019) em 12\% dos estabelecimentos de alimentação fora do lar não possuíam loja física, trabalhando exclusivamente por meio de entregas por aplicativo, sem portas abertas para o público no ano de 2019.

A pesquisa constatou que $81,3 \%$ dos entrevistados trabalham com o delivery, tal fato corrobora com o descrito por Dutra \& Zani (2020), que afirma que após o início da pandemia de covid-19, houve um aumento quase que imediato de $30 \%$ nos pedidos de alimentos por delivery, somente no mês de março/2020 no Brasil. Segundo Teixeira e Mourão (2021) cerca de 34,3\% dos consumidores começaram a usar aplicativos de entregas de alimentos associados a situação sanitária excepcional e houve um aumento de 71,6\% na frequência dos pedidos de entrega, promovendo um acréscimo das quantidades de resíduos sólidos urbanos, gerando um alerta para a gestão dos municípios e as organizações sociais envolvidas no recolhimento destes resíduos.

Dentre os segmentos dos participantes da pesquisa constatou-se que 37,4\% dos entrevistados trabalham em docerias; 31,7 \% com cozinha brasileira; 22,8\% como lanchonete e 14,6\% em pizzaria. Essas informações representam a transformação vivida pelo delivery e pela popularização da alimentação fora do lar nas últimas três décadas no Brasil, tendo como principal consequência a perda de saúde pelo consumidor, que vem sendo afetada negativamente, visto que a composição nutricional dos alimentos e bebidas disponíveis no delivery, deve ser semelhante à daqueles presentes nos estabelecimentos físicos, que no Brasil tem como característica um perfil nutricional alto em calorias e pobre em nutrientes. A aquisição de comida por delivery pode contribuir para o consumo de alimentos não saudáveis e para o desenvolvimento de doenças crônicas não transmissíveis 
(Keeble et al., 2020; Botelho et al., 2020).

O serviço de delivery para $48 \%$ dos entrevistados, começou apenas depois do lockdow e casos da pandemia no Brasil, corroborando com o que diz Dutra e Zani (2020) que relataram em seu estudo, um crescimento significativo nesse tipo de serviço durante a pandemia de SARS-CoV-2. Esse aumento imediato trouxe consequências para os estabelecimentos, conforme constatado na pesquisa, onde $53,7 \%$ dos entrevistados afirmaram que precisaram fazer algum tipo de modificação/alteração no seu estabelecimento para poder utilizar o serviço de entrega.

Tal fato se relaciona diretamente com a afirmação que $48 \%$ dos entrevistados iniciaram o serviço depois da pandemia e, portanto, o seu espaço físico não havia sido pensado/desenvolvido para associar o funcionamento físico com o serviço de entrega. Serviço este que passou também a ser utilizado pelas classes C e D com a popularização dos preços (Iodice, 2019) e pela necessidade de vender sem que os espaços físicos estivessem abertos ao público, em diferentes períodos durante a pandemia.

A entrega dos alimentos nos estabelecimentos é variada e busca alternativas para utilizar os aplicativos de delivery, sendo distribuída entre funcionários próprios, parentes e até mesmo o dono do negócio. Tal fato deve se dar por conta das tax as altas cobradas por aplicativos de delivery, com valores de taxas chegando a $30 \%$ do valor total do pedido, que em momentos de crise e de necessidade passaram a ser absorvidos pelo próprio custo de funcionamento do restaurante (Botelho et al., 2020).

De acordo com a RDC 91 (Brasil, 2001) define que embalagem para alimentos é o artigo que está em contato direto com os alimentos, destinado a contê-los, desde a sua fabricação até a sua entrega ao consumidor, com a finalidade de protegêlos de agente externos, de alterações e de contaminações, assim como de adulterações. Segundo Azeredo (2012), as embalagens desempenham um papel fundamental na indústria alimentícia graças às suas múltiplas funções, versatilidade e garantia de proteção sanitária.

Além de conter o produto, a embalagem é muito importante na sua conservação, mantendo qualidade e segurança sanitária, atuando como barreira contra fatores responsáveis pela deterioração química, física e microbiológica, que confere proteção ao alimento e há vários materiais autorizados pelos órgãos competentes, para o uso adequado para cada grupo de alimentos (ANVISA, 2020; Bernardo et al., 2015).

Constatou-se que os estabelecimentos já utilizavam o modelo delivery, antes da pandemia, empregando os seguintes materiais de embalagens: 65,9\% utilizavam plástico; 48,8\% isopor; 39\% papel cartonado e 30,9\% alumínio. Tal informação é preocupante, visto que a maioria desses materiais são contaminantes persistentes no meio ambiente. Segundo Silva et al. (2013) o modelo econômico em que vivemos, vem causando mudanças e grandes impactos negativos ao meio ambiente por meio das ações antrópicas. Tais alterações estão relacionadas principalmente ao aumento da população, incentivo e elevação do consumo, globalização e inovações tecnológicas para prolongamento da vida útil, através do uso de embalagens para os alimentos.

Dentre esses impactos da industrialização, destacam-se a disposição inadequada dos resíduos sólidos, principalmente os materiais de origem plástico. Na Malásia, apenas nos primeiros dias de quarentena, a geração de resíduos sólidos domiciliares aumentou quase 30\% (WMAM, 2020). Lembrando que os polímeros sintéticos empregados para alimentos são em sua maioria, de alta taxa de degradação (Macedo et al., 2020).

O alto volume de utilização de materiais plásticos como embalagens de delivery é alarmante. Os produtos plásticos fazem parte do dia-dia de bilhões de pessoas. São produzidas mais de 400 milhões de toneladas por ano no mundo. A palavra "plástico" diz respeito a um grupo de materiais sintéticos feitos a partir de hidrocarbonetos, que são formados através da polimerização por meio de reações químicas utilizando matérias orgânicas que contém carbono derivadas do petróleo bruto e gás natural (Almeida, 2021). Em função da taxa de degradação lenta, os plásticos causam um grande impacto no meio ambiente, pois, permanecem na natureza por longos anos até que sejam decompostos por fatores abióticos e microrganismos 
decompositores de polímeros (Macedo et al., 2020; Silva et al., 2021).

Tais informações associadas ao fato de que, atualmente, não existe reciclagem de plástico, apenas reciclagem do ciclo aberto ou subciclagem (downcycling). Cada vez que um pedaço de plástico é reciclado, sua qualidade é degradada. O plástico pode ser reciclado apenas um certo número de vezes antes de acabar no incinerador ou aterro. Sendo assim, o que consideramos reciclagem do plástico significa, na verdade, apesar postergar o descarte final para proteção das futuras gerações no planeta (Atlas do plástico, 2020).

A utilização do isopor em grande quantidade, como é no caso das embalagens em alimentos e como foi constatado na pesquisa apresenta um problema até agora não resolvido. $\mathrm{O}$ isopor é um termoplástico moldado com o seu aquecimento e expandido com o poliestireno. Por ser um plástico celular rígido, sustentável, sem risco para a saúde e para o meio ambiente, o seu processo de reutilização pode ser infinito podendo até voltar a condição de matéria prima, não perdendo suas propriedades mecânicas. É 100\% reciclável e 100\% atóxico, usa um processo de reciclagem que consome poucos recursos naturais (energia e água) e gera poucos resíduos, porém a realidade da reciclagem do isopor, está longe de ser a ideal no Brasil, com apenas $34 \%$ do isopor reciclado, o percentual vem aumentando com o passar dos anos, porém ainda está muito aquém do ideal frente a crescente utilização em serviços de alimentação no Brasil (Morais \& Vidigal, 2021; Oliveira et al., 2021).

A má gestão dos resíduos sólidos urbanos leva a sérios problemas ambientais, tais como: contaminação de solos, das águas superficiais e subterrâneas e do ar; danos para fauna e flora; combustão nociva de gases; proliferação de vetores e ocupação de grandes espaços nas cidades pelos resíduos, que em sua grande maioria são persistentes quanto a degradação natural e, portanto, classificados como fortes poluentes ambientais (MMA, 2018).

Quando questionados sobre a continuidade no uso das embalagens de delivery durante a pandemia, 73,2\% dos entrevistados afirmaram continuar utilizando os mesmos materiais, esbarrando no mesmo problema ambiental supracitado. Como alento a esta realidade, 15,4\% informaram que adotaram o uso de papel cartonado, um produto mais agradável e que agride menos o meio ambiente. Aqui não se pode confundir a embalagem de papel cartonado para delivery com a embalagem de papel cartonado para longa vida, como as de leite UHT, pois segundo Pereira et al. (2020) estas são difíceis de reciclar por conta de sua composição que envolve seis camadas diferentes (plástico, papel e alumínio), que associadas conferem proteção do alimento.

Em contrapartida, em 10,6\% dos entrevistados informaram que passaram a utilizar o plástico como embalagem, o que segundo Bernardo et al. (2015) pode acarretar problemas de saúde, pois alguns plastificantes que fazem parte da composição das embalagens, são considerados disruptores endócrinos (compostos químicas que alteram o bom funcionamento do sistema hormonal), interferem no conjunto de reações do metabolismo, denominados de anabolismo e catabolismo, ligação ou eliminação dos hormônios naturais, responsáveis pela manutenção do equilíbrio e regulação dos processos de manutenção do metabolismo basal.

Os motivos para a troca da embalagem nos estabelecimentos que apontaram que o fizeram foi principalmente por iniciativa do próprio estabelecimento, com $92,1 \%$ por ser uma alternativa mais barata ou como uma estratégia de marketing para vendas, a depender do público do estabelecimento. Apesar da modificação das embalagens, $81,1 \%$ dos consumidores foram indiferentes às mudanças, pavimentando o discurso de mudança por conta da redução de custo. Cerca de 5,4\% dos clientes demonstraram insatisfação com as mudanças, um fator apontado como o principal motivo dessa insatisfação foi o de que as embalagens apresentavam menor resistência, chegando muitas vezes amassadas ou quebradas e com o alimento exposto.

Outro fator de insatisfação, foi o de interferência de sabor, muitas vezes causada pelo material de menor qualidade ou inadequado para determinado tipo de produto que muitas vezes e colocado a temperaturas elevadas. Segundo Campbell-Platt (2015), tal fator pode ser consequência da interação das embalagens com o alimento acondicionado, levando a migração de 
compostos tóxicos e alteradores de sabor para os alimentos, podendo assim causar danos à saúde do consumidor, uma vez que essa migração química se constitui um risco para a integridade física dos comensais.

Um fator relevante para que os clientes estivessem indiferentes pode ter sido porque em 52,5\% dos estabelecimentos que modificaram as embalagens não alteraram o preço final dos produtos, não impactando em custo econômico para o cliente. Dentre esses estabelecimentos quase $80 \%$ informaram que não precisaram fazer adaptações nas quantidades ou qualidade dos insumos para manter o preço final, o que reforça a tese de que as substituições visavam a troca de embalagens por outras mais baratas ou de mesmo valor, porém com qualidade inferior. Visto que o preço médio das embalagens sofreu um reajuste por conta da alta demanda instantânea advinda do momento pandêmico, conforme apontado pelos estabelecimentos na pesquisa, que constatou que $91,9 \%$ dos entrevistados perceberam um aumento no preço das embalagens. Em contrapartida, 45,8\% dos estabelecimentos que modificaram as embalagens tiveram um aumento no preço final do produto, repassado ao consumidor.

As embalagens são adquiridas em $75,6 \%$ em lojas físicas enquanto os outros $24,4 \%$ adquiriram de forma virtual, tal informação pode ser em decorrência do perfil dos estabelecimentos pesquisados, a maioria é de pequenas empresas, com fluxo de caixa menor e portanto sem a possibilidade de fazer grandes compras e de estocar esse material em grande quantidade, o que endossa a aquisição em lojas físicas, que dispensam custos extras, como o do frete e atendem aquela demanda imediata, sem a necessidade de aguardar a chegada do material de aquisição virtual. Tal informação coaduna com o fato de $63,4 \%$ dos estabelecimentos comprarem de fornecedores locais e $24,3 \%$ de fornecedores regionais. Esses percentuais também demonstram os motivos pelos quais a maioria dos estabelecimentos $(83,7 \%)$ usam embalagens prontas disponíveis no comércio local, isto é, sem uso de personalização do estabelecimento nas embalagens.

Quando indagados sobre a relação entre a escolha da embalagem com o impacto para o meio ambiente, houve dissonância do que foi analisado com o que foi respondido, possivelmente pelo fato de que uma empresa ambientalmente responsável, ser bem quista no mercado e pelos clientes, ou até pela falta de conhecimento do impacto que as embalagens de delivery podem causar no meio ambiente. Cerca de 48,7\% dos entrevistados afirmaram levar em consideração o impacto ambiental da embalagem escolhida por eles, porém o antagonismo existe quando mais de 65,9\% utilizam plástico e 48,8\% utilizam isopor, elementos de difícil decomposição natural no meio ambiente ou da carência de métodos de reciclagem no país.

\section{Considerações Finais}

Os estabelecimentos pesquisados apresentaram um aumento na oferta de delivery, com alternativas para diminuição dos custos com o serviço, como a adoção de entregadores particulares, como funcionário e familiares, além da adoção de embalagens de menor custo para o estabelecimento, ainda que de maior impacto ambiental. Apesar do constatado pela pesquisa, quase a metade dos entrevistados declara que se preocupa com o impacto das embalagens no meio ambiente, demonstrando que há necessidade de melhor discussão e participação da sociedade civil e poder público, quanto a temática de uso de embalagens descartáveis para alimentos.

Os resultados apresentados neste trabalho podem servir de incentivo para pesquisas mais abrangentes e mais aprofundadas sobre o tema, que seriam de grande contribuição para ratificar as informações que constam no estudo e promover mudanças na forma de se consumir alimentos prontos, por meio de delivery, a fim de mitigar os problemas com resíduos sólidos gerados em práticas de consumo alimentar.

\section{Referências}

ABRASEL, Associação brasileira de bares e restaurantes. (2019). Delivery movimenta R \$11 bilhões por ano. https://abrasel.com.br/noticias/noticias/deliverymovimenta-r-11-bilhoes-por-ano-enquanto-franquias-de-alimentacao-diversificam-a-oferta-de-produtos/.

Almeida, S. L. de. (2021). Consumo de embalagens plásticas em serviço de nutrição de um hospital universitário: avaliação de consumo antes e durante pandemia por covid-19. Universidade Federal do Rio Grande do Sul - UFRGS. 
ANVISA. (2020). Materiais em contato com alimentos. Gerência-Geral De Alimentos. Gerência de Avaliação de Risco e Eficácia de Alimentos. $5^{\text {a } e d i c ̧ a ̃ o ~}$ Brasília, 06 de março de 2020 .

Atlas do plástico. (2020) Fatos e números sobre o mundo dos polímeros sintéticos. Fundação Heinrich Böll. 978-65-87665-02-3. Novembro.

Azeredo, H. M. C. (2012). Fundamentos de Estabilidade de Alimentos. Embrapa, 326p.

Bernardo, P. E. M., Navas, S. A., Murata, L. T. F., \& de Alcântara, M. R. D. S. (2015). Bisfenol A: o uso em embalagens para alimentos, exposição e toxicidade-Uma Revisão. Revista do Instituto Adolfo Lutz, 74(1), 1-11.

Botelho, L. V., Cardoso, L. de O., \& Canella, D. S. (2020). COVID-19 e ambiente alimentar digital no Brasil: reflexões sobre a influência da pandemia no uso de aplicativos de delivery de comida. Cadernos de Saúde Pública 36(11). https://doi.org/10.1590/0102-311X00148020.

BRASIL. (2004). Resolução da diretoria colegiada - RDC No 216, de 15 de Setembro de 2004. Dispõe sobre "Regulamento Técnico de Boas Práticas para Serviços de Alimentação" constante do Anexo desta Resolução. Órgão Emissor: ANVISA- Agência Nacional de Vigilância Sanitária. https://bvsms.saude.gov.br/bvs/saudelegis/anvisa/2004/res0216_15_09_2004.html>

Campbell-Platt, G. (2015). Ciência e Tecnologia de Alimentos. Manole, 536p.

Dutra, J. A. A., \& Zani, R. (2020) Uma análise das práticas de delivery de alimentos em tempos de pandemia do COVID-19. Almanaque Multidisciplinar de Pesquisa, 7(2).

Gil, A. C. (2002). Como elaborar projetos de pesquisa. (4a ed.), Atlas.

Iodice, G. (2019) Conheça o bilionário mercado de entregas de comida. https://forbes.com.br/negocios/2019/06/conheca-o-bilionario-mercado-de-entregas-decomida/.

Keeble, Adams, J., Sacks, G., Vanderlee, L., White, C. M., Hammond, D., \& Burgoine, T. (2020). Use of Online Food Delivery Services to Order Food Prepared Away-From-Home and Associated Sociodemographic Characteristics: A Cross-Sectional, Multi-Country Analysis. International Journal of Environmental Research and Public Health, 17(14), 5190. https://doi.org/10.3390/ijerph17145190

Macedo, I. M. E., de Souza, M. D. L. C., Shinohara, N. K. S., dos Santos, C. S., \& da Silva, M. K. G. (2020). Reciclagem do Polietileno Tereftalato (PET) no Fomento da Economia Circular. Brazilian Journal of Development, 6(8), 57704-57723.

MMA, Ministério do Meio Ambiente. (2018). Logística Reversa - SINIR - Sistema Nacional de Informações sobre a Gestão de Resíduos Sólidos. https://sinir.gov.br/logistica-reversa

Morais, M. O. \& Vidigal, H. (2021). O processo de logística reversa aplicado no produto EPS (ISOPOR). Research, Society and Development, 10(2), e52910212908-e52910212908.

Moreira, A. G., da Mota, N. R., Pereira, D. M., Santa Rosa, V. A. C., \& Pires, C. A. A. (2022). Aspectos clínicos e epidemiológicos de pacientes com COVID19 atendidos em um centro de referência na Amazônia. Conjecturas, 19(1).

Novaes, A. A., Alencar, M. C., Araújo, C. D. S. A., \& Boleta-Ceranto, D. D. C. F. (2020). Percepção de alunos concluintes de odontologia sobre o impacto da pandemia do covid-19 no futuro profissional. Cenários odontológicos em tempos de pandemia, 221.

Oliveira, W. R. M. D., \& Rocha, L. M. da S. (2021). Abordagem da tipologia e aplicações de reciclagem de eps's. Revista Multidisciplinar de Educação e Meio Ambiente, 2(4), 20. 10.51189/rema/2700. https://editoraime.com.br/revistas/index.php/rema/article/view/2700.

Pereira, M. E., Junior, J. F. M., \& Bianchi, R. M. da C. (2020) Embalagem cartonada: metodologia para separação e reciclagem de seus componentes. 1-388416.

Ruggiero, A. S. (2021). Dirigindo: emergência, fresta ou frustração? Revista ARA, 10(10), 159-175. https://doi.org/10.11606/issn.2525-8354.v10i10p159-175

Silva, A. L. P., Prata, J. C., Walker, T. R., Duarte, A. C., Ouyang, W., Barcelò, D., \& Santos, T. R (2021). Increased plastic pollution due to COVID-19 pandemic: Challenges and recommendations. Chemical Engineering Journal, 405 (126683).

Silva, C. O., Santos, G. M., \& Silva, L. N. (2013). A degradação ambiental causada pelo descarte inadequado das embalagens plásticas: estudo de caso. Revista Eletrônica em Gestão, educação e tecnologia ambiental, 13(13), 2683-2689.

Teixeira, K. L., \& Mourão, F. V. (2021). O descarte de embalagens de delivery em tempos de pandemia. Interação, 21(1), 1-13.

WMAM, Waste Management Association of Malaysia. (2020). The following Interview was prepared by ISWA and Completed by ISWA's National Member in Malaysia, The Waste Management Association of Malaysia (WMAM). IWSA -International solid waste association. 4 p. 